\title{
Interstellar Methanol from the Lab to Protoplanetary Disks
}

\author{
Maria N. Drozdovskaya ${ }^{1}$, Catherine Walsh $^{1}$, Ruud Visser ${ }^{2}$, \\ Daniel Harsono ${ }^{3}$ and Ewine F. van Dishoeck ${ }^{1,4}$ \\ ${ }^{1}$ Leiden Observatory, Leiden University, \\ P.O. Box 9513, 2300 RA, Leiden, The Netherlands \\ email: drozdovskaya@strw.leidenuniv.nl \\ ${ }^{2} \mathrm{ESO} ;{ }^{3}$ University of Heidelberg; ${ }^{4} \mathrm{MPE}$
}

Keywords. astrochemistry, stars: formation, (stars:) planetary systems: protoplanetary disks

\section{Summary}

Interstellar methanol is thought to be the precursor of larger, more complex organic molecules. It holds a central role in many astrochemical models (e.g., Garrod \& Herbst 2006). Methanol has also been the focus of several laboratory studies (e.g., Watanabe et al. 2004, Fuchs et al. 2009), in an effort to gain insight into grain-surface chemistry, which potentially builds chemical complexity already in the cold, dark prestellar phase. The case of methanol is a prime example of experimental work having implications on astronomical scales. Drozdovskaya et al. (2014) unified physical and chemical models to simulate infalling material during the birth of a low-mass protostar. An axisymmetric 2D semi-analytic collapse model (Visser et al. 2009), wavelengthdependent radiative transfer calculations with RADMC3D (Dullemond \& Dominik 2004) and a comprehensive gas-grain chemical network (Walsh et al. 2014) were used to study two modes of protoplanetary disk formation. One mode predominantly grows the disk via viscous spreading, while the other by the continuous infall of matter. Drozdovskaya et al. (2014) conclusively showed that the abundance and distribution of methanol in the disk is determined by the infall path, leading to regions in which methanol is enhanced and/or depleted relative to what is expected for static models of protoplanetary disks. The results for the comet-forming zone show a level of depletion for methanol commensurate with that observed towards cometary comae (e.g., Mumma \& Charnley 2011) showing that chemical processing en route from protostar to protoplanetary disk is important for setting the composition of comet- and planet-building material. The ties between extrasolar systems and Solar System bodies are now being tested by cometary data from the Rosetta mission and protoplanetary disk observations by ALMA (Drozdovskaya et al. in prep.).

\section{References}

Watanabe, N., Nagaoka, A., Shiraki, T., \& Kouchi, A. 2004, ApJ, 616, 638

Garrod, R. T. \& Herbst, E. 2006, A\& A, 457, 927

Dullemond, C. P. \& Dominik, C. 2004, A\&\&A, 417, 159

Fuchs, G. W., Cuppen, H. M., Ioppolo, S., et al. 2009, A\& $A$, 505, 629

Visser, R., van Dishoeck, E. F., Doty, S. D., et al. 2009, A\& $A$, 495, 881

Walsh, C., Millar, T. J., Nomura, H., et al. 2014, A\&AA, 563, A33

Drozdovskaya, M. N., Walsh, C., Visser, R., et al. 2014, MNRAS, 445, 913

Drozdovskaya, M. N., Walsh, C., van Dishoeck, E. F., et al. 2015b, MNRAS, in prep.

Mumma, M. J. \& Charnley, S. B. 2011, ARA\&A, 49, 471 